\title{
Editorial: special issue on extremes in finance
}

\author{
Paul Embrechts
}

Published online: 9 November 2014

(C) Springer Science+Business Media New York 2014

Extreme, synchronized rises and falls in financial markets occur infrequently, but they do occur. The problem with the models is that they did not assign a high enough chance of occurrence to the scenario in which many things go wrong at the same time - the 'perfect storm' scenario. (Business Week, September 1998)

With globalization increasing, you'll see more crises. Our whole focus is on the extremes now - what's the worst that can happen to you in any situation because we never want to go through that again. (John Meriwether, Wall Street Journal, 21 August 2000)

Both quotes relate to the LTCM case in August 1998, the downfall of an illustrious hedge fund with serious repercussions throughout the wider financial industry. The more recent financial crisis of 2007-2009 unfortunately produced ample of examples of the way in which extreme events in financial markets can raise havoc throughout the world economy. In a very dramatic way, the problems on Wall Street hit Main Street, and this in full force. As a direct effect, practitioners, clients, regulators, politicians as well as academics have become increasingly sensitized on the importance of a basic understanding of how to engage with, how to model, how to protect against, and most importantly, how to communicate extreme phenomena and their consequences. In this special issue we concentrate on extremes in finance, though the title should be understood more widely as 'extremes in finance, insurance and economics'.

From a teaching point of view, Extreme Value Theory (EVT) has entered the level of finance and insurance - 101, i.e. it has become basic course material for more quantitatively oriented students in the fields of insurance, finance and economics. For academia, and this over a wide ranging area of applications, EVT based research

P. Embrechts ( $ه)$

RiskLab, Department of Mathematics, ETH Zurich, CH-8092 Zurich, Switzerland

e-mail: embrechts@math.ethz.ch 
is both obtaining interesting questions from finance and insurance, and at the same time is able to offer useful tools and techniques for a better understanding of socalled non-normal behavior. Embrechts et al. (1997) and McNeil et al. (2005) offer textbook treatments of relevant material. The present special issue offers a glimpse of some relevant research going on in this highly fruitful interplay between different areas of methodological and applied research.

I have tried to bring together papers which highlight the variety of ways in which the modeling of extremal events enters the broader world of finance. As a consequence, the papers not only reflect econometric applications of EVT at the level of univariate and multivariate tail-behavior, but also concentrate on topics like portfolio optimization, risk aggregation, as well as the statistical and numerical estimation of regulatory risk measures (like Value-at-Risk (VaR), a high-quantile based risk measure). In this context, the analysis of model uncertainty, in particular at the level of interdependence, plays an increasingly important role in day-to-day business and regulation.

Rare are the fields of application where an EVT related quantity is written in the law as a quantity to be statistically estimated; VaR is such a case. In its recent publication, Basel (2012), the Basel Committee on Banking Supervision formulated the following question: 'What are the likely constraints with moving from Value-atRisk (VaR) to Expected Shortfall (ES), including any challenges in delivering robust backtesting and how might these be best overcome?' Similar discussions take place in the realm of insurance regulation under the Solvency 2 regime. The above question not only raised a considerable amount of reactions from the industry, but also from academia; for the latter, see for instance Embrechts et al. (2014). As the question involves statistical estimation of risk measures at quantile levels beyond $99 \%$, EVT becomes a canonical tool in some of the ensuing discussions. I very much hope that this special issue of Extremes will contribute to getting more researchers involved in discussions around these themes: such research is not only academically challenging but at the same time of high societal relevance!

In realizing this special issue, I would not only like to thank the contributing authors for their stimulating publications, but also the many anonymous referees who, in a timely fashion, helped considerably in keeping the project on track.

\section{References}

Basel: Consultative document May 2012. Fundamental review of the trading book. Basel Committee on Banking Supervision. Bank for International Settlements, Basel (2012)

Embrechts, P., Klüppelberg, C., Mikosch, T.: Modelling Extremal Events for Insurance and Finance. Springer, Berlin (1997)

Embrechts, P., Puccetti, G., Rüschendorf, L., Wang, R., Beleraj, A.: An academic response to Basel 3.5. Risks 2(1), 25-48 (2014)

McNeil, A.J., Frey, R., Embrechts, P.: Quantitative Risk Management: Concepts, Techniques, Tools. Princeton University Press, Princeton (2005) 